\title{
Relación entre el uso de antipsicóticos y riesgo de insuficiencia respiratoria aguda en pacientes con EPOC
}

Relationship between the use of antipsychotics and risk of acute respiratory failure in COPD patients.

\section{Comentado de:}

Wang MT, et al. JAMA Psychiatry. 2017;74(3):252-260.PMID: $28055066^{1}$.

\section{Objetivo}

Determinar si el uso de antipsicóticos está asociado al aumento de riesgo de insuficiencia respiratoria aguda (IRA) en pacientes con enfermedad pulmonar obstructiva crónica (EPOC).

\section{Diseño, lugar y pacientes}

Estudio poblacional de casos-cruzados, realizado en Taiwán, República de China. Incluyó pacientes con IRA diagnosticados en el hospital o en centros de atención de emergencia, identificados a través de la Base de Datos de Investigación del Seguro Nacional de Salud de dicha región que requirieron intubación o ventilación mecánica, entre el 1 de enero de 2000 y el 31 de diciembre de 2011.

\section{Evaluación de los factores de riesgo}

Se excluyeron del análisis los pacientes que presentaban factores de riesgo que constituían en sí mismos el origen etiológico de IRA. Con respecto a posibles variables confundidoras, se realizó una comparación entre ambos grupos; aquellas variables en las que hubo diferencias entre los grupos (por ejemplo, falla cardiaca, neumonía, cáncer, agentes antiplaquetarios, etc.) fueron incluidas en el análisis multivariado de riesgo para el consumo de antipsicóticos y el desarrollo de IRA.

\section{Medición de resultados principales}

Se evaluó riesgo de IRA; el uso de antipsicóticos se comparó con sí mismo durante los días 1 a 14 (el período de riesgo según los informes de casos anteriores) y los días 75 a 88 (período de control) anteriores al evento e IRA o fecha índice. También se examinaron la clase de antipsicóticos, la vía de administración y la dosis.

\section{Resultados principales}

Entre los 61.620 pacientes con EPOC, 5.032 desarrollaron IRA con una media de edad 74,4 años (DE 9,9), siendo 3.533 hombres $(70,2 \%)$.

A 590 pacientes con IRA $(11,7 \%)$ se les prescribió al menos un antipsicótico durante el período de caso, comparado con 443 $(8,8 \%)$ durante el período de control correspondiente a 1,66 veces de riesgo ajustado de IRA, independientemente de la clase farmacológica y la vía de administración.

Se identificó un riesgo de IRA dependiente de la dosis del antipsicótico (prueba de tendencia, OR ajustado 1,35; IC 95\% 1,19 a 1,52; $P<0,001$ ), que aumentó de 1,52 veces el riesgo con una dosis diaria baja, a un riesgo de 3,74 veces para una dosis alta (ver Tabla 1 ).

Tabla 1. Riesgo de insuficiencia respiratoria aguda asociado al uso de antipsicóticos. Notas: OR: odds ratio. *Ajuste según admisión en unidad de cuidados intensivos, insuficiencia cardíaca, neumonía, asma, cáncer, uso de agentes antiplaquetarios, inhibidores de la enzima convertidora de angiotensina, betabloqueantes, antihistamínicos de primera generación, benzodiacepinas y no benzodiacepinas, antidopaminérgicos, opioides, anestésicos, antiespasmódicos, anticolinérgicos, anti-inflamatorios no esteroides, drogas que provocan fallo respiratorio, amiodarona, metilxantinas, medicaciones para EPOC, número de visitas a emergencias, y de internaciones por EPOC y número de prescripciones de antibióticos y corticoides en pacientes ambulatorios. ${ }^{* \star D D D: ~ d o s i s ~ d e f i n i d a ~ d i a r i a . ~}$

\begin{tabular}{|l|c|c|c|}
\hline Característica & Casos & Control & OR ajustado* \\
\hline & $\begin{array}{c}(\mathrm{n}=5.032) \\
\mathrm{n}, \%\end{array}$ & $\begin{array}{c}(\mathrm{n}=5.032) \\
\mathrm{n}, \%\end{array}$ & $\begin{array}{c}\text { (IC } 95 \%) \\
\text { Uso de antipsicóticos }\end{array}$ \\
$\begin{array}{l}590(11,7) \\
\begin{array}{l}\text { Dosis baja antipsicóticos } \\
\text { (< o igual 25 DDD) }\end{array}\end{array}$ & $383(7,6)$ & $299(5,9)$ & $1,52(1,20$ a 1,92) \\
$\begin{array}{l}\text { Dosis alta antipsicóticos } \\
\text { (> 1.00 DDD) }\end{array}$ & $43(0,9)$ & $23(0,5)$ & $3,74(1,68$ a 8,36) \\
Antipsicóticos típicos & $310(6,2)$ & $202(4)$ & $1,70(1,31$ a 2,19) \\
\hline
\end{tabular}

\section{Conclusiones}

El uso de antipsicóticos en pacientes con EPOC se asocia con un incremento de riesgo agudo y dependiente de la dosis de desarrollar IRA. Los médicos deben tener cuidado al prescribir antipsicóticos a pacientes con EPOC y evitar en lo posible el uso de altas dosis.

Conflicto de interés de los autores: Sin conflicto de intereses. 


\section{Comentario}

Las conclusiones del presente artículo son coincidentes con publicaciones anteriores, en las cuales la asociación entre el uso de antipsicóticos e IRA ya era señalada ${ }^{2,3}$.

En la actualidad, el uso de antipsicóticos no se limita exclusivamente al tratamiento de esquizofrenia u otras patologías del espectro de las psicosis. También se indican como fármacos de primera línea (monoterapia o adyuvancia) en otros cuadros como depresión, trastornos afectivos bipolares, trastornos conductuales, etc.

En el caso de que a un paciente con EPOC, de cierta evolución, se le hubiera indicado recientemente el uso de medicación antipsicótica ${ }^{4}$, el médico generalista debería considerar el riesgo señalado a fin de poder realizar una evaluación minuciosa, intentando pesquisar posibles signos o síntomas que evidencien una exacerbación del cuadro pulmonar de base del paciente (con posible desarrollo de IRA); sobre todo atendiendo al tiempo transcurrido desde la instauración del psicofármaco, la dosis al momento de la instauración y aumentos posteriores.

Si bien la clase del antipsicótico (típicos o atípicos, según el mecanismo de acción y perfil clínico de efectos adversos) no parece representar una variable de peso, se vio que la posibilidad de que estos pacientes presenten IRA es mayor en las primeras semanas desde la administración del psicofármaco, como así también si dicho medicamento se ha administrado en dosis altas desde el inicio. Asimismo, y en relación a la dosificación, el riesgo aumentaría con el incremento de la misma. Por último, en lo referido a las vías de administración del antipsicótico, no se especifica cuáles se encuentran incluidas dentro de señaladas como "cualquier inyección", ya que se podría considerar, por un lado, la vía intramuscular para un cuadro agudo de excitación psicomotriz, como así también la administración de medicación antipsicótica de depósito para un cuadro mental crónico ${ }^{4}$, con propiedades farmacocinéticas diferentes en cada caso; y quizás también con diferencias en la probabilidad de desarrollo de IRA en estos pacientes.

\section{Conclusiones del comentador}

El uso extendido de fármacos antipsicóticos invita a tener presente los resultados y datos mencionados a fin de integrarlos en la práctica cotidiana, de manera de poder detectar tempranamente cualquier signo de exacerbación respiratoria o signos de IRA en pacientes con EPOC que hayan recientemente iniciado o incrementado un tratamiento con medicación antipsicótica.

Tomás Abudarham [ Servicio de Psiquiatría, Hospital Italiano de Buenos Aires. tomas.abudarham@hospitalitaliano.org.ar ]

Aburdaham T. Relación entre el uso de antipsicóticos y riesgo de insuficiencia respiratoria aguda en pacientes con EPOC. Evid Actual Pract Ambul. 2019;22(4):e001048. Comentado de: Wang MT, et al. Association Between Antipsychotic Agents and Risk of Acute Respiratory Failure in Patients With Chronic Obstructive Pulmonary Disease. JAMA Psychiatry. 2017; 74(3):252-260. doi:10.1001/jamapsychiatry.2016.3793. PMID: 28055066

\section{Referencias}

1. Wang M, Tsai C, Lin CW, Yeh C, Wang Y, Lin H. Association Between Antipsychotic Agents and Risk of Acute Respiratory Failure in Patients With Chronic Obstructive Pulmonary Disease. JAMA Psychiatry. 2017;74(3):252-260.

2. Stahl SM. Stahl's essential psychopharmacology: neuroscientific basis and practical application. and others, editor. Cambridge University Press; 2013. 4th ed. EEUU.

3. Schatzberg AF. Essentials of clinical psychopharmacology. and others, editor. APA Publishing; 2013. 3rd ed. EEUU.

4. Tiihonen J. Real-World Effectiveness of Antipsychotic Treatments in a Nationwide Cohort of 29823 Patients With Schizophrenia". JAMA Psychiatry. 2017;74(7):686-693. 